\title{
OBSERVATION ET SUIVI DE DEFORMATIONS DE SURFACE D'ORIGINE ANTHROPIQUE PAR INTERFEROMETRIE RADAR SATELLITAIRE
}

\author{
Daniel Raucoules ${ }^{1}$, Elisabeth Simonetto ${ }^{2}$, Bénédicte Fruneau ${ }^{3}$ \\ 1: BRGM DRP/RAI, 3 avenue C. Guillemin, 45060, Orléans, France, d.raucoules@brgm.fr \\ 2 : GeF, CNAM/ESGT, 1 Bd Pythagore, 72000 Le Mans, France, elisabeth.simonetto@lecnam.net \\ 3 : Université Paris-Est, LaSTIG, IGN, UPEM, 5 bd Descartes, 77454 Marne-la-Vallée, France, benedicte.fruneau@u-pem.fr
}

\begin{abstract}
Résumé
L'activité humaine relative aux travaux souterrains (mines, construction de réseaux souterrains) ou à l'injection/extraction de fluides (eau, sel, hydrocarbures) induit des phénomènes qui peuvent se traduire par des déplacements du terrain en surface. Ces mouvements du sol sont généralement suivis par des techniques in situ. L'interférométrie différentielle d'images radar satellite offre une alternative et apporte des mesures complémentaires aux méthodes de surveillance classique. Cet article propose une synthèse sur les champs d'application et les apports de cette méthode de télédétection pour la détection, la cartographie et la surveillance de tels phénomènes anthropiques en les illustrant avec des exemples.
\end{abstract}

Mots-clés : Interférométrie radar différentielle, imagerie satellite, déformation du sol, phénomènes d'origine anthropique

\begin{abstract}
Ground deformation phenomena can occur due to human activity related to underground works (mines, underground network construction) or the injection/extraction of fluids (water, salt, hydrocarbons). These ground movements are usually surveyed by in situ techniques. Differential interferometry of satellite radar images is an alternative and offers complementary measurements to the conventional monitoring methods. Through several examples, this article proposes an overview of application domains and contributions of this remote sensing technique for the detection, mapping and monitoring of such anthropic-due phenomena.
\end{abstract}

Keywords : Differential InSAR, satellite imagery, ground deformation, anthropic phenomena

\section{Introduction}

De nombreux phénomènes anthropiques, qu'il s'agisse de travaux souterrains (notamment miniers) ou d'extractions de fluides (pétrole, eau, gaz) du sous-sol, se traduisent en surface par de la déformation du sol. Ces phénomènes pouvant affecter les personnes, les bâtiments et les réseaux, il est nécessaire de mettre en place un suivi des déformations de surface pour prévenir les risques associés. Des moyens de surveillance peuvent par ailleurs être exigés auprès d'un exploitant dans le cadre de règlementations spécifiques. Outre la surveillance, les mesures de déformation du sol peuvent entrer dans le cadre d'analyses de risques. II s'agira dans ce cas de préciser la position et l'extension de la zone soumise à déformation et d'examiner son évolution passée (en détectant notamment une augmentation éventuelle des vitesses de déformation observées) afin d'évaluer sa dangerosité future.

L'interférométrie SAR (radar à synthèse d'ouverture), ou InSAR, est une technique de mesure du terrain par imagerie radar (voir par exemple Massonnet et Feigl, 1998). La fin des années 90 marque le début de l'utilisation de I'InSAR différentielle, ou DInSAR, pour la détection et le suivi de mouvements verticaux (subsidence ou soulèvement) liés à des phénomènes d'origine anthropique. En milieu urbain, Amelung et al. (1999) ont détecté une large subsidence sur Las Vegas suite à des pompages en eau. Des déformations de plus faible extension et liées à des travaux souterrains ont été observés sur Naples (Tesauro et al., 2000) et sur Paris (Fruneau et al., 1998). En zone non urbaine, Carnec et Delacourt (2000) se sont intéressés à la déformation induite par l'activité minière sur le site Gardanne. Auparavant, seules les mesures au sol (GNSS, nivellement, etc.) permettaient de quantifier les déplacements.

Désormais, l'utilisation de l'interférométrie différentielle s'est généralisée, et s'envisage de manière systématique pour le suivi de phénomènes anthropiques. Cette technique vient en général compléter les mesures au sol, parfois les remplacer.

Elle se démarque des autres sources de mesures de déplacements par la couverture spatiale qu'elle offre, et qui reste inégalée; sa spécificité est de mieux circonscrire la zone de déformation en déterminant son extension spatiale. De même, pour ce qui est de l'échantillonnage spatial, la densité de mesure est en général supérieure. Couverture spatiale, échantillonnage (spatial et temporel) et précision des mesures sont fonction de plusieurs facteurs comme par exemple le type de capteur radar, tandis que les limites d'utilisation dépendent aussi de l'objet mesuré (Simonetto et al., 2019).

D'une manière générale, l'interférométrie différentielle permet de répondre au besoin de fournir des informations 
sur la déformation en surface afin de mieux comprendre le phénomène sous-jacent. Ceci sera utile par exemple pour estimer - à l'aide d'une modélisation numérique de la déformation- les paramètres et caractéristiques du processus de déformation en profondeur qui reproduisent au mieux la déformation observée.

Dans certains cas, seules les mesures interférométriques peuvent être exploitées: il arrive souvent que les mesures au sol ne soient mises en place que postérieurement à la constatation de conséquences visibles telles que des désordres sur le bâti. Par conséquent, l'interférométrie différentielle - du fait de la possibilité de valoriser des données d'archives remontant parfois à plusieurs années avant l'observation de dégâts - constitue la seule source d'information sur l'évolution passée d'un phénomène (Colesanti et al., 2005). L'intérêt est donc de détecter des mouvements mal connus et permettre de mieux les caractériser (géométrie de la déformation et évolution temporelle passée).

En ce qui concerne la surveillance de sites ou infrastructures soumis à des aléas impliquant une déformation de surface, l'interférométrie différentielle peut jouer un rôle important, mais il est peu envisageable de l'utiliser directement en tant que système d'alerte: l'échantillonnage temporel de mesure accessible avec les capteurs existants est actuellement insuffisant (y compris à partir de capteurs de répétitivité meilleure que la semaine tels que COSMO-SkyMed). Outre l'échantillonnage temporel, le temps de traitement des données reste une limitation dans le cadre d'un système d'alerte, pour lequel la durée entre l'acquisition de la donnée et la transmission de l'alerte est cruciale.

Toutefois, les observations interférométriques fournissent des informations qui peuvent être associées à des mesures au sol, qui sont en général ponctuelles. En effet, les données au sol fournissent un meilleur échantillonnage temporel et un accès à l'information qui peut être immédiat, mais elles ne permettent généralement pas de caractériser le phénomène dans toute sa complexité spatiale. L'interférométrie différentielle fournira ainsi une information complémentaire (mise à jour régulièrement) ne serait-ce que pour optimiser le réseau au sol par rapport à la géométrie de la déformation. L'interférométrie différentielle servira à implanter les points de mesure là où il y a réellement de la déformation, tout en garantissant que les points de référence du réseau soient effectivement stables, mais aussi à répartir les points de mesure de telle sorte qu'ils fournissent une bonne représentation spatio-temporelle de la déformation (par exemple en densifiant le réseau là où l'interférométrie différentielle a mis en évidence les gradients de déformation les plus importants), tout en limitant leur nombre au strict nécessaire (Loschetter et al., 2015). Compte tenu de la diversité des caractéristiques spatiales et temporelles des déformations d'origine anthropique (extensions typiquement de quelques mètres à quelques kilomètres, et durées allant de l'heure à des dizaines d'années), les différentes techniques interférométriques présentent un intérêt, que ce soit la méthode conventionnelle ou les méthodes avancées telles que l'interférométrie sur réflecteurs persistants (PSI) présents naturellement sur la zone d'étude, ou issus de coins réflecteurs installés. De même, les diverses caractéristiques (répétitivité, résolution spatiale, fréquence du signal) des différents capteurs existants peuvent être choisies selon les différents types de déformations et contextes. Par exemple, des zones urbanisées mais aussi des zones à couvertures végétalisées importantes peuvent être affectées par un phénomène de déformation de surface. Suivant les cas, différents choix sur la fréquence du signal radar et la répétitivité des acquisitions peuvent être opérés.

Dans cet article, nous illustrons l'utilisation de l'interférométrie différentielle par quelques exemples où la technique a fourni des résultats intéressants, tout d'abord en zone urbanisée (partie 2), dans le contexte minier (partie 3) et enfin sur des sites de stockage et d'exploitation de fluides (partie 4).

\section{Application de l'interférométrie différentielle en zone urbanisée}

L'intérêt de la détection et du suivi des phénomènes de déformation du sol en zone urbaine réside à la fois sur la présence d'enjeux importants (bâtiments et personnes vulnérables à ce type de phénomènes), et de la diversité des causes anthropiques et naturelles pouvant produire ces déformations. Du point de vue des techniques interférométriques elles-mêmes, l'avantage est que celles-ci s'avèrent généralement performantes en présence d'un couvert urbanisé du fait de la faible décorrélation temporelle (Zebker et Villasenor, 1992). Dans le but de caractériser ces aléas, l'intérêt de l'interférométrie différentielle vient de sa capacité à cartographier la déformation à l'échelle de l'agglomération dans sa globalité permettant ainsi de détecter et suivre ce type de phénomène à coût raisonnable par rapport à des mesures au sol. La figure 1 montre un exemple couvrant l'agglomération de Paris, mettant en évidence des déformations liées à la présence de cavités souterraines (carrières), tassements de remblais, et travaux souterrains (Magalhaes, 2010). L'interférométrie différentielle permet également un suivi à une échelle plus fine, atteignant l'échelle du bâtiment. La figure 2 montre la déformation subie par le Grand Palais au cours de la période 1995-2001 et due à des instabilités affectant ses fondations (Raucoules et al., 2007). 
Parmi les différentes sources de déformation du sol en zone urbaine, on ne s'intéressera ici qu'à deux phénomènes particuliers d'origine anthropique. D'une part, aux mouvements liés à l'extraction de fluide et d'autre part à ceux dus aux travaux souterrains.
Leurs caractéristiques en termes de vitesses et d'étendues spatiales de la déformation diffèrent. D'autres causes notamment d'origine naturelle, telles que le retrait gonflement, les glissements de terrain, les mouvements d'origine sismo-tectonique, le tassement de remblai, la

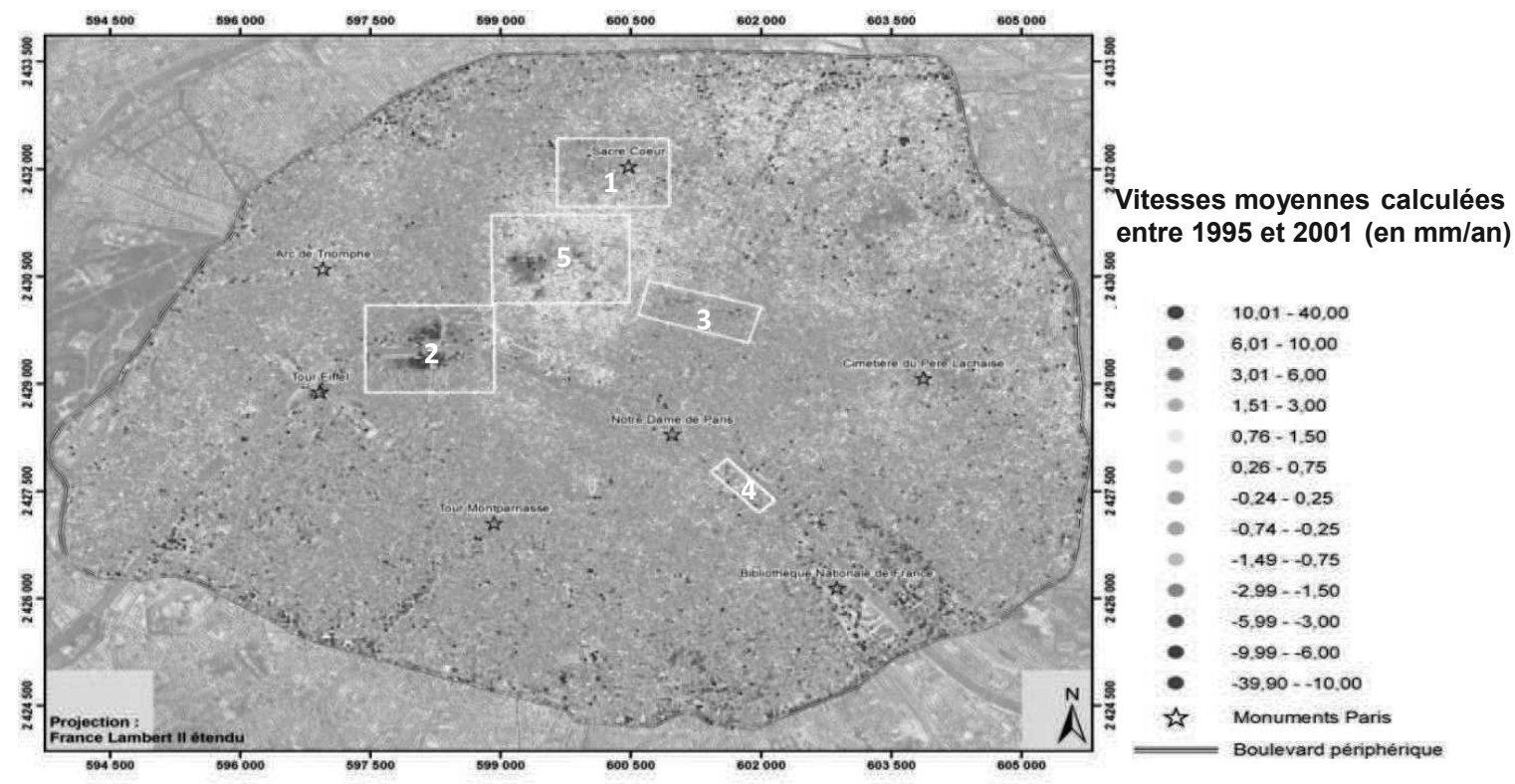

Figure 1: Carte des vitesses moyennes des déplacements (dans la ligne de visée du capteur radar) calculées entre 1995 et 2001 par PSI sur la ville de Paris à partir d'images ERS et Envisat.

Différents secteurs, présentant des contextes variés, affichent des déplacements $d u$ sol, pour certains significatifs, d'origines diverses: (1) la butte Montmartre (dus à la présence d'anciennes carrières), (2) la zone Grand-Palais/Invalides (liés à la proximité de la Seine et de formations géologiques particulières), (3) les buttes Bonne Nouvelle et Saint Martin (dus à la présence de remblais), (4) le quai Saint Bernard (en rive gauche de la Seine), (5) le secteur Opéra/Saint-Lazare (dus à l'arrêt des pompages conséquents). D’après (Magalhaes, 2010).

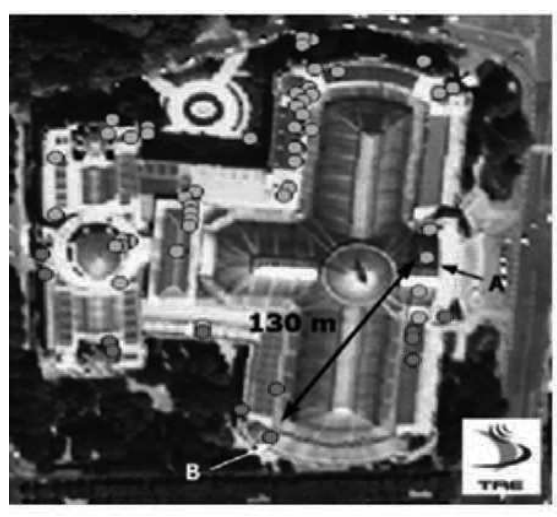

Average PS LOS velocity

- $\quad-2,55--0,64 \mathrm{~mm} /$ year

- $-0,63--0,56 \mathrm{~mm} / \mathrm{year}$
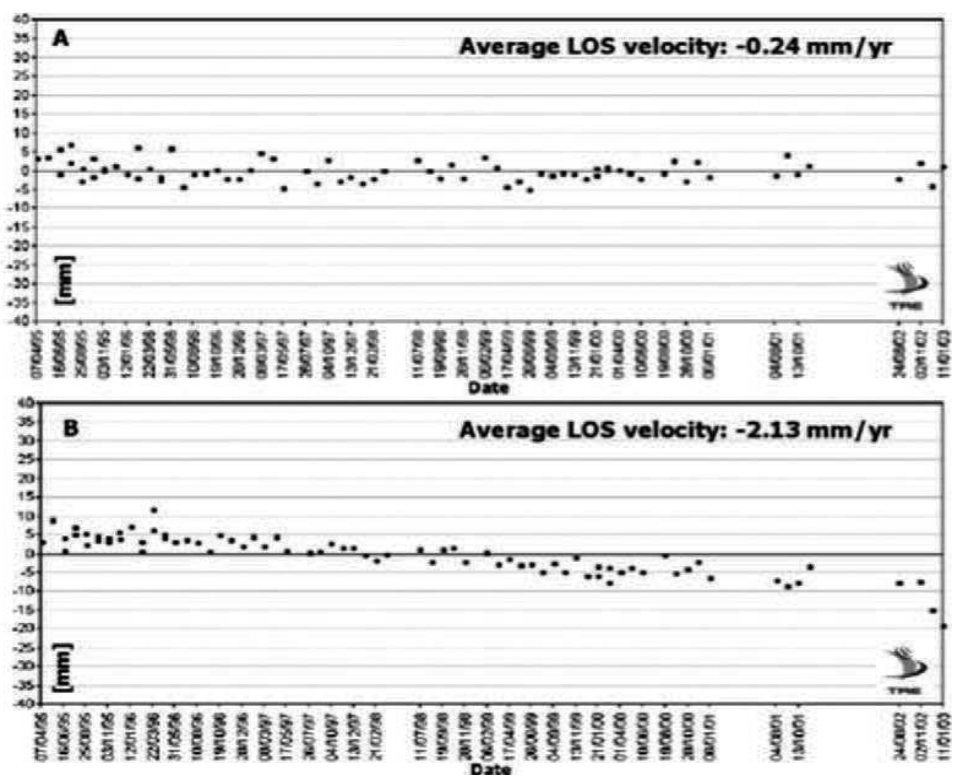

Figure 2 : Déformation sur le Grand Palais, d'après Raucoules et al (2007). A gauche, localisation des points PS et vitesses moyennes estimées dans la ligne de visée du capteur radar (LOS). A droite, évolution temporelle du mouvement pour deux points, le point A apparaissant stable sur la période étudiée entre avril 1995 et janvier 2003, et le point B montrant un phénomène de tassement qui peut être expliqué par une instabilité des fondations (dégradation de pieux en bois). 
dissolution de gypse ou sel, l'effondrement de cavités peuvent affecter les zones urbanisées. Toutefois, leur origine n'étant pas nécessairement anthropique, ils ne font pas l'objet principal de cet article.

L'interférométrie différentielle n'est pas adaptée à tous les phénomènes de déformation du sol en zone urbaine. Dans de nombreux cas - mais heureusement pas de manière systématique - les phénomènes se produisent avec des vitesses fluctuantes, souvent de durée limitée à quelques mois ou quelques années et sont peu étendus (moins de quelques centaines de mètres). Les déformations résultant d'effondrements de cavités (carrières, marnières, cavités naturelles) ou de certains travaux souterrains posent par exemple des difficultés du point de vue de la mesure interférométrique car les gradients de déformations trop importants peuvent entraîner une perte d'information (voir l'exemple de la figure 6). Ainsi, l'interférométrie différentielle fournit des informations utiles pour des déformations modérées et d'extensions bien supérieures à la taille du pixel de l'image SAR, ce qui ne permet toutefois pas de couvrir l'intégralité du spectre des phénomènes de déformation du sol pouvant impacter une zone urbaine.

En ce qui concerne l'extraction de fluides, le processus peut induire un phénomène de subsidence, tandis que l'injection quant à elle peut produire une remontée de la surface. La croissance de la demande en ressources en eau résultant du développement des agglomérations au cours du $20^{\text {ème }}$ siècle a eu ainsi pour conséquence des phénomènes de subsidences de grande ampleur. En effet, la baisse du niveau dans les nappes souterraines due aux pompages peut entraîner en fonction des spécificités locales du sous-sol une subsidence plus ou moins forte. Plusieurs exemples passés d'application de l'interférométrie différentielle concernent des phénomènes de subsidence urbaine liée à l'extraction d'eau souterraine pour répondre au besoin croissant de la population: la subsidence sur la ville de Mexico au Mexique (Strozzi et Wegmuller, 1999 ; Yan et al, 2012) en est un exemple très connu avec une déformation importante pouvant atteindre $40 \mathrm{~cm} / \mathrm{an}$. Les villes de Djakarta en Indonésie (Chaussard et al., 2013) ou de Manille aux Philippines (Raucoules et al., 2013) présentent également des déformations très significatives (>10 cm/an) de même nature. Outre les pompages nécessaires pour alimenter la population en eau, ceux liés aux besoins de l'industrie et l'agriculture peuvent également être à l'origine de ce type de phénomène. A titre d'exemple, sur la ville de Prato en Italie, l'interférométrie différentielle a permis de mettre en évidence des mouvements de l'ordre de $10 \mathrm{~cm} / \mathrm{an}$ (Raucoules et al., 2003). Quelle que soit l'origine du phénomène, la mesure de déformation en surface permet ou pourrait permettre à l'utilisateur final (autorités locales ou exploitant d'une ressource) de mieux anticiper les répercussions des pompages et rectifier le processus d'extraction.

Les travaux souterrains quant à eux provoquent des déformations en surface qui peuvent être la conséquence directe de création de vides ou résulter des pompages nécessaires aux travaux. Ce type de déformations a récemment été suivi par $\mathrm{PSI}$ sur les travaux de creusements de tunnels ferroviaires sur la ville de Bologne (Italie) par l'équipe de TRE ALTAMIRA ${ }^{1}$ mettant en évidence des subsidences et soulèvements de l'ordre du cm/an. Des phénomènes similaires (subsidences et soulèvements) ont aussi été révélés sur les villes de Rennes (Nahli et al., 2018) suite à la construction de la ligne $b$ du métro urbain (Figure 3), ou de Lyon (Thierry et al., 2005) lors de la construction de l'autoroute urbaine TEO (Figure 4), ou encore, sur un cas plus ancien, à proximité de la gare Saint Lazare à Paris (Figure 5) lors des travaux de construction de la gare du RER E (Fruneau et Sarti, 2000 ; Le Mouelic et al., 2002, Fruneau et al., 2004).

\section{Application de l'interférométrie différentielle au cas de l'exploitation de mines souterraines}

L'activité minière, présente ou passée, se traduit souvent par des déformations de la surface du sol. Dans ce contexte, le suivi des mines souterraines par DInSAR présente un intérêt particulier dans la mesure où la déformation peut affecter - au-delà des infrastructures de l'exploitant - d'autres éléments situés à la surface tels que bâtiments, réseau, infrastructures, etc... En France, les dernières mines souterraines (il s'agissait de houillères) ont été fermées en 2004. Aussi le suivi de la déformation de surface s'inscrit dans une perspective de gestion de risque après-mine.

Suivant le type d'exploitation (par chambres et piliers, par foudroyage, etc...), la répartition de la déformation de surface entre la période d'activité de la mine et l'aprèsmine peut varier. A titre d'exemple, dans le cas d'une exploitation par foudroyage (la cavité est volontairement effondrée à l'arrière du front d'exploitation), la déformation s'effectue en trois étapes - des détails peuvent être trouvés par exemple dans (Gueguen et al., 2009) - : un affaissement initial, correspondant à $10-15 \%$ de la déformation totale (pendant moins d'un mois), une phase d'affaissement principal de plusieurs mois, d'environ $80 \%$ de la déformation totale (avec des vitesses de déformation atteignant plusieurs $\mathrm{dm} / \mathrm{an}$ ) et enfin un affais-

\footnotetext{
${ }^{1}$ http://treuropa.com/construction-engineering/tunneling
} 


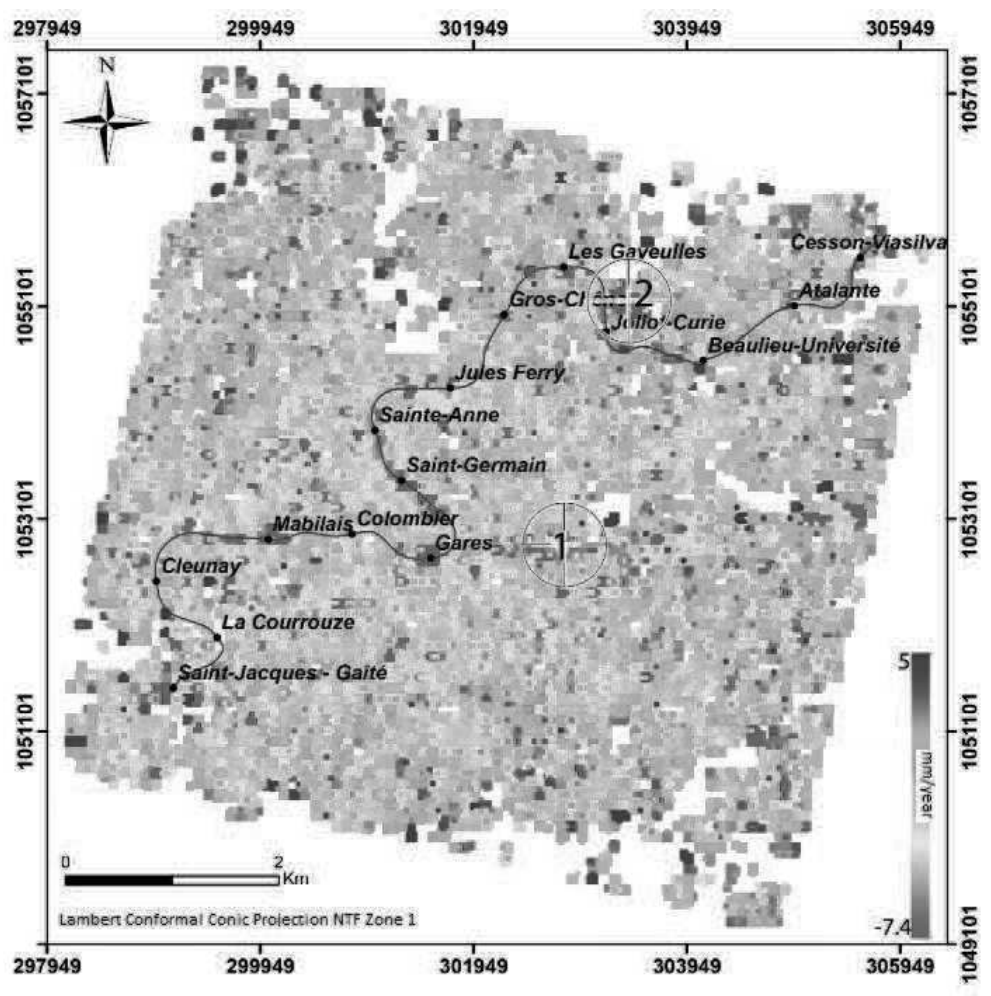

Figure 3: Carte des déplacements moyens (dans la ligne de visée du capteur) en mm/an sur la ville de Rennes, produite avec des images Sentinel-1 entre octobre 2014 et avril 2017. Le tracé de la ligne de métro $b$ est représenté en noir. Les travaux d'excavations ont débuté au sud de la ligne en janvier 2015, pouvant causer des mouvements horizontaux et verticaux du sol. On observe des subsidences sur certaines stations. D’après (Nahli et al., 2018).

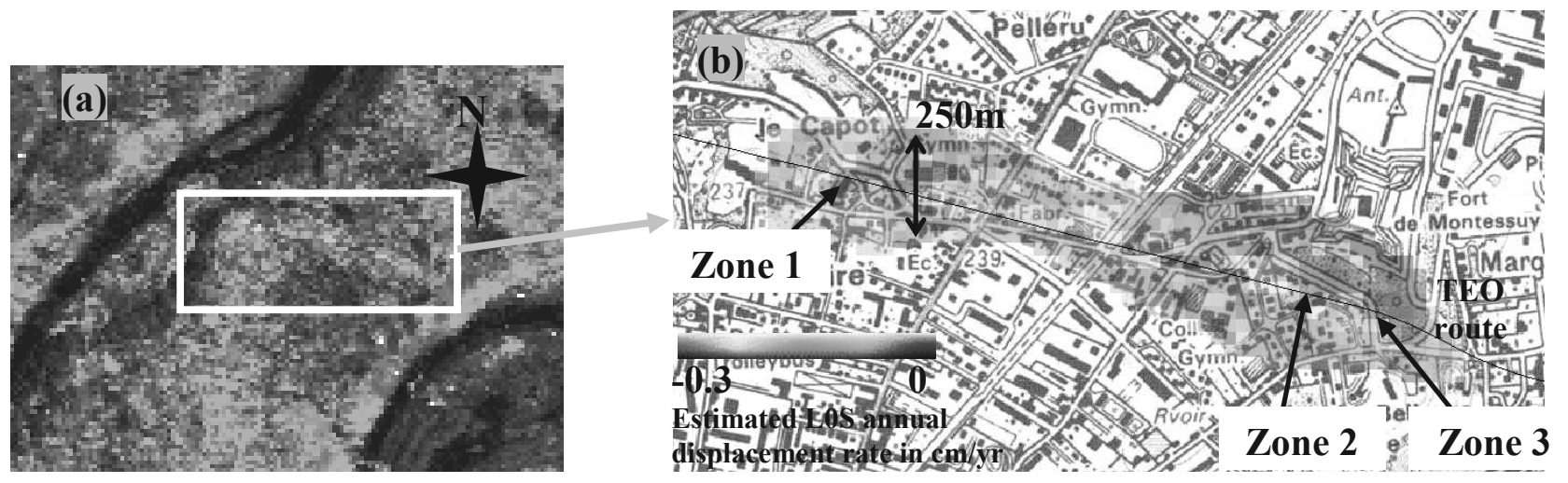

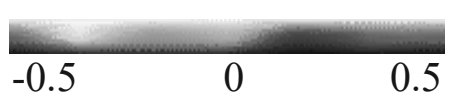

Estimated LOS annual displacement rate in $\mathrm{cm} / \mathrm{yr}$ (c)

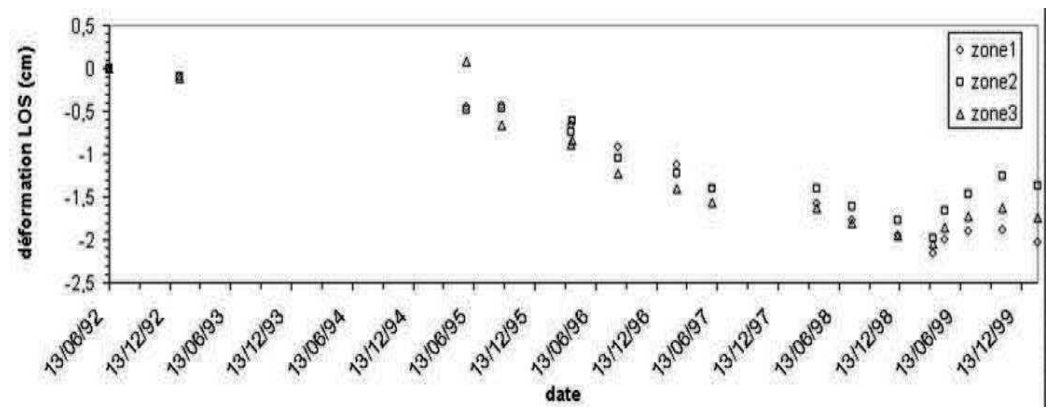

Figure 4 : (a) Image des déplacements moyens (dans la ligne de visée du capteur) en cm/an sur un quartier de la ville de Lyon, produite avec des images ERS-1 et ERS-2 entre juillet 1992 et janvier 2000. On observe une subsidence de forme linéaire (en jaune, de largeur $250 \mathrm{~m}$ et de longueur $1300 \mathrm{~m}$ centrée autour de la position 45,79 ; 4,84\%). (b) Zone de mouvement superposée sur la carte IGN. Le phénomène s'explique par le creusement de l'autoroute souterraine TEO représentée par un trait noir et la géologie du terrain. (c) Les séries temporelles des déplacements montrent un tassement de 0,4 cm/an sur les trois zones 1, 2 et 3 entre 1994 et 1999 puis un rebond, probablement lié à la fin des travaux. D'après (Thierry et al., 2005). 


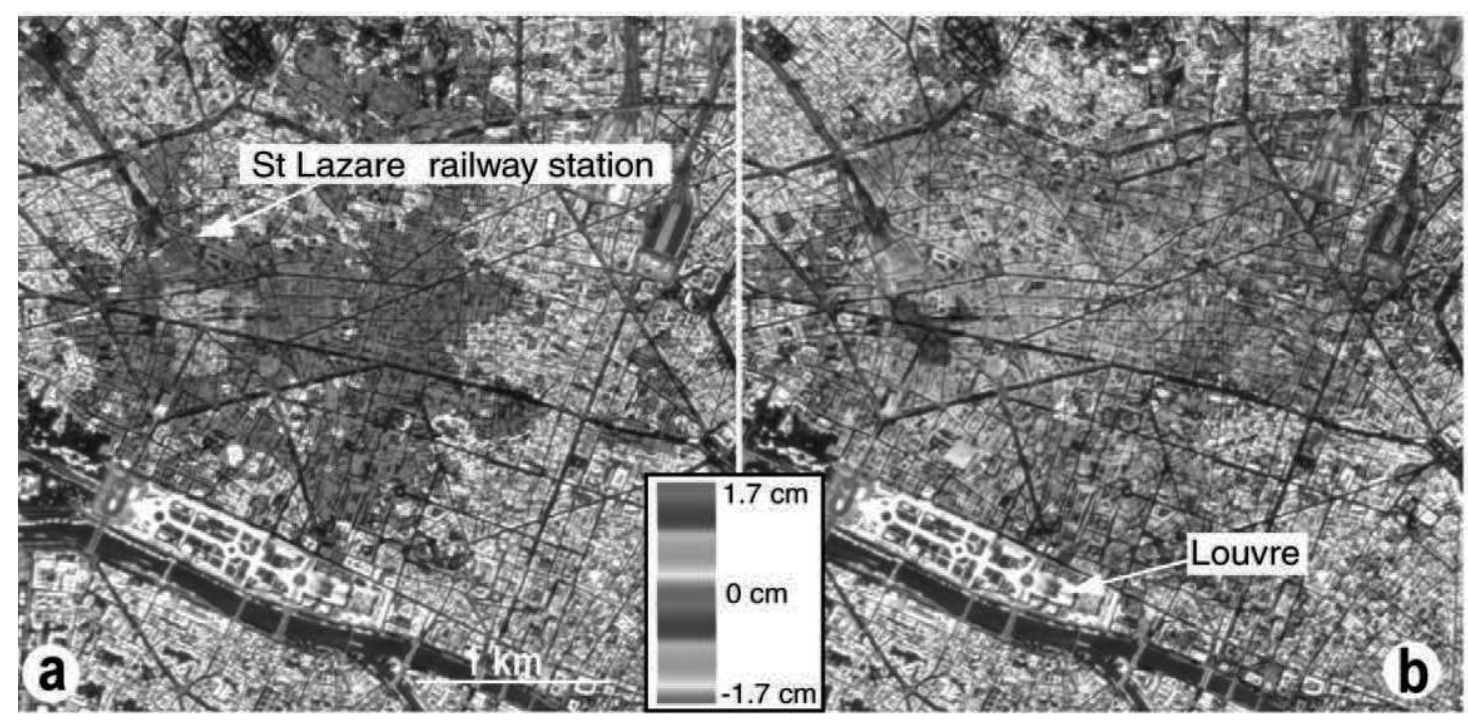

Figure 5 : Déformations mesurées par DInSAR (converties en déplacements verticaux) avec des images ERS sur le secteur SaintLazare (Paris) liées aux pompages en eau lors de la construction de la gare Haussmann Saint-Lazare du RER E. (a) Carte de subsidence totale due au début des pompages en eau, de mai 1994 à avril 1995. (b) Carte de la remontée totale due à la fin des pompages et à la lente recharge de la nappe, de juin à octobre 1998. D'après (Fruneau et al., 2004).

-sement résiduel, correspondant à $5-10 \%$ de la déformation totale et qui s'étale sur plusieurs années. Spatialement, la déformation observée s'étend un peu audelà de la taille du panneau (soit 250 à 500 m).

Lorsque les contextes des sites à surveiller sont favorables à l'utilisation des techniques interférométriques (si l'amplitude de la déformation maximale n'est pas trop importante et si l'occupation du sol le permet, c'est-à-dire avec peu de couvert végétal), la cartographie de la déformation durant la phase active du site peut être effectuée (e.g., Carnec et Delacourt, 2000 ; Engelbrecht et al., 2011). Dans certaines conditions, en particulier lorsque la déformation est significative, un suivi de la déformation résiduelle est également possible (Raucoules et al., 2009).

Pour les exploitations par chambres et piliers (où de la roche exploitable est laissée en place afin de constituer les piliers supportant le toit), la déformation est plus limitée lors de l'exploitation elle-même et difficile à appréhender. Toutefois, avec la remontée du niveau de l'eau souterraine après la fermeture de la mine et l'arrêt des pompages nécessaires lors de l'exploitation, les piliers peuvent se dégrader à très long terme, et les caractéristiques mécaniques des remblais changer. Cet ennoyage peut alors se traduire par des phénomènes de déformation de surface très variables (de quelques $\mathrm{mm}$ jusqu'à plusieurs mètres) suivant les sites et être observés sur plusieurs années (Colesanti et al., 2005, Gueguen et al., 2009).

La figure 6 montre un exemple de déformation aprèsmine sur le site de Roncourt (Lorraine) où un affaissement important a eu lieu en 1999. La déformation au centre de la cuvette à la suite de cet affaissement est trop importante pour être vue par interférométrie différentielle. Toutefois, les observations en bord de cuvette ont permis de mettre en évidence l'évolution temporelle de la déformation et en particulier le début du déplacement 10 mois avant la constatation des premiers dégâts sur les bâtiments et la mise en place d'un réseau de suivi au sol en 1999 .

En ce qui concerne les mines à ciel ouvert en cours d'exploitation, les mesures interférométriques de type PSI présentent un intérêt pour la surveillance de la stabilité des talus et gradins nécessaire au fonctionnement de l'exploitation. La figure 7 présente un exemple de suivi d'une mine de charbon, exploitation à ciel ouvert et souterraine, dans le nord de l'Espagne (Mora et al., 2013).

\section{Application de l'interférométrie différentielle au suivi de l'exploitation et du stockage de fluides autres que les ressources en eau (gaz naturel, $\mathrm{CO}_{2}$, pétrole, sel par dissolution, etc.)}

L'injection de gaz à des fins de stockage à des profondeurs importantes (plusieurs centaines de mètres), que ce soit du gaz naturel ou du dioxyde de carbone (stockages géologiques de $\mathrm{CO}_{2}$ dans le cadre de la réduction d'émissions de gaz à effet de serre dans l'atmosphère) produit des soulèvements du sol à proximité du point d'injection. De façon similaire, l'extraction entraîne une subsidence. Les déformations résultant de ces processus sont généralement très faibles (allant de la fraction de $\mathrm{mm} / \mathrm{an}$ à quelques $\mathrm{cm} / \mathrm{an}$ ). En ce qui concerne les stockages de gaz naturel, les déformations peuvent être cycliques en raison d'une alter- 

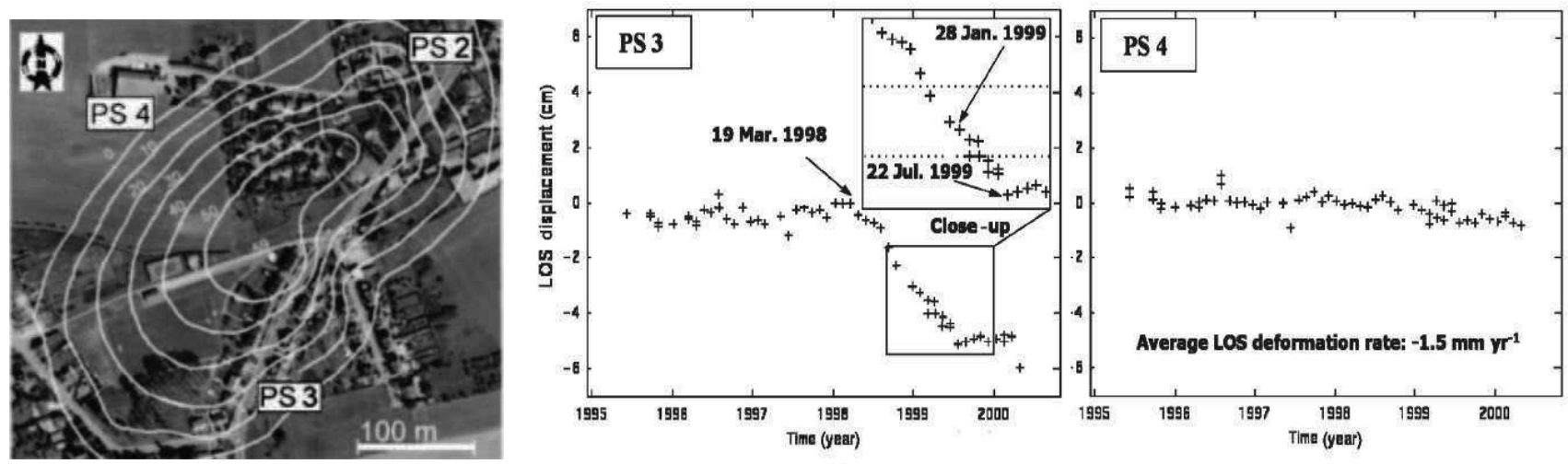

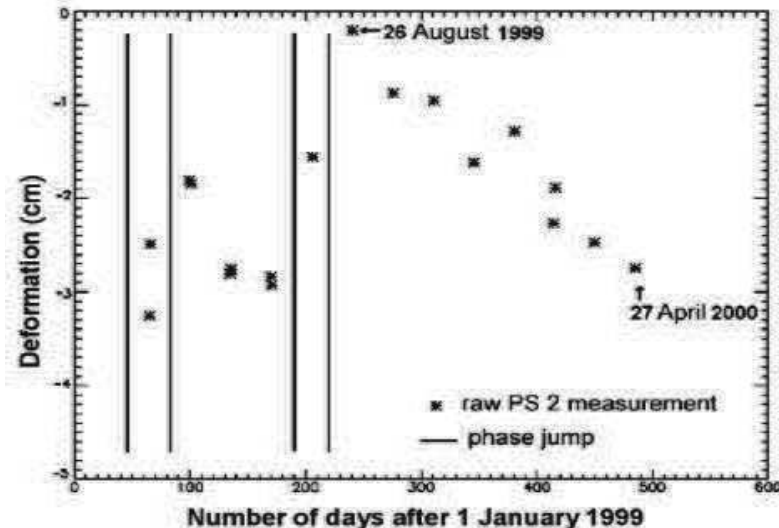

(a)

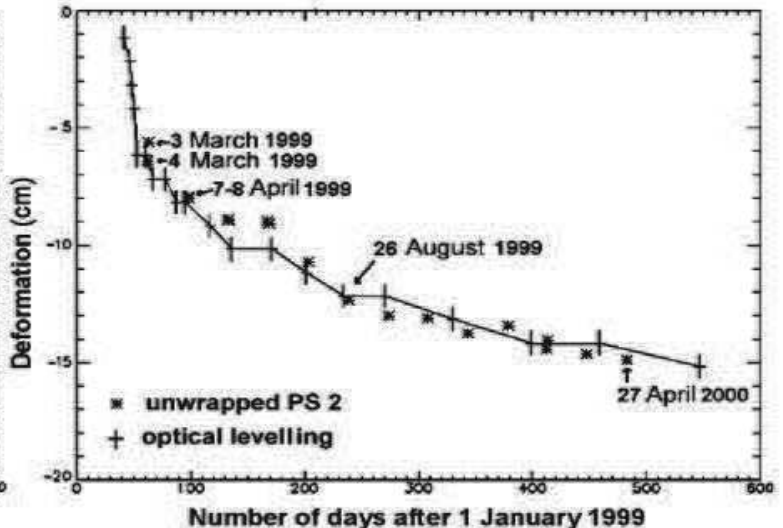

(b)

Figure 6 : Déformation sur Roncourt par méthode PSI, site ferrifère ayant été exploité jusqu'au début des années 90 et ayant subi des désordres en surface postérieurement à l'arrêt de son exploitation, d'après (Colesanti et al., 2005). En haut à gauche : position de trois PS situés à proximité de la zone affectée et, en jaune, courbes de l'affaissement cumulé (en cm) estimé à partir de mesures de nivellement acquises en 1994 et 1999. Le déplacement de $59 \mathrm{~cm}$ au centre de la cuvette est attribué à un affaissement mesuré à partir de février 1999. En haut à droite : séries temporelles des déplacements dans la ligne de visée du capteur des points PS3 et PS4. PS3 montre que le mouvement a débuté en 1998. En bas à gauche : série temporelle du déplacement pour PS2. Sur la figure de gauche, on observe des ambiguïtés résiduelles de la mesure interférométrique à cause du mouvement important (phénomène de repliement du signal interférométrique). Sur la figure de droite, après correction de ces ambiguïtés, la mesure interférométrique de PS2 est concordante avec celle du nivellement.

-nance injection/extraction et être confondues avec des phénomènes naturels de retrait-gonflement. $\mathrm{Vu}$ ces caractéristiques, l'interférométrie différentielle peut s'avérer peu performante pour ce type d'application, d'autant que ces installations sont généralement situées sur des sites avec des occupations du sol non urbain où les phénomènes de décorrélation temporelle seront plus importants. Néanmoins, compte tenu de l'importance qu'il y a à suivre ce type d'installation industrielle, l'application de l'interférométrie différentielle a fait (et fait toujours) l'objet d'études. On citera en particulier, le cas du démonstrateur d'injection de $\mathrm{CO}_{2}$ d'In Salah (Algérie). Ce site d'extraction de gaz naturel a servi pour une série de tests d'injection de $\mathrm{CO}_{2}$ entre 2004 et 20112. La technique DInSAR (PSI et conventionnelle) a permis la cartographie de la déformation de surface résultant de l'injection (Vasco et al., 2010 ; Onuma et Ohkawa, 2009). Sur la période suivie, des vitesses de soulèvement de plus de $5 \mathrm{~mm} / \mathrm{an}$ dues à l'activité des différents puits d'injection sont mises en évidence (Figure 8, Rucci et al., 2011).

Par ailleurs l'asymétrie des géométries des déformations observées en surface a pu être interprétée par la présence de failles. II faut toutefois relativiser cette performance de la technique, dans la mesure où il s'agit d'une zone désertique (donc très favorable à l'interférométrie) et dont les caractéristiques du sous-sol ont pour effet de produire une déformation significative comparée à d'autres sites d'injection envisagés par rapport au taux d'injection (Rohmer et Raucoules, 2012).

\footnotetext{
${ }^{2}$ http://www.insalahco2.com
} 


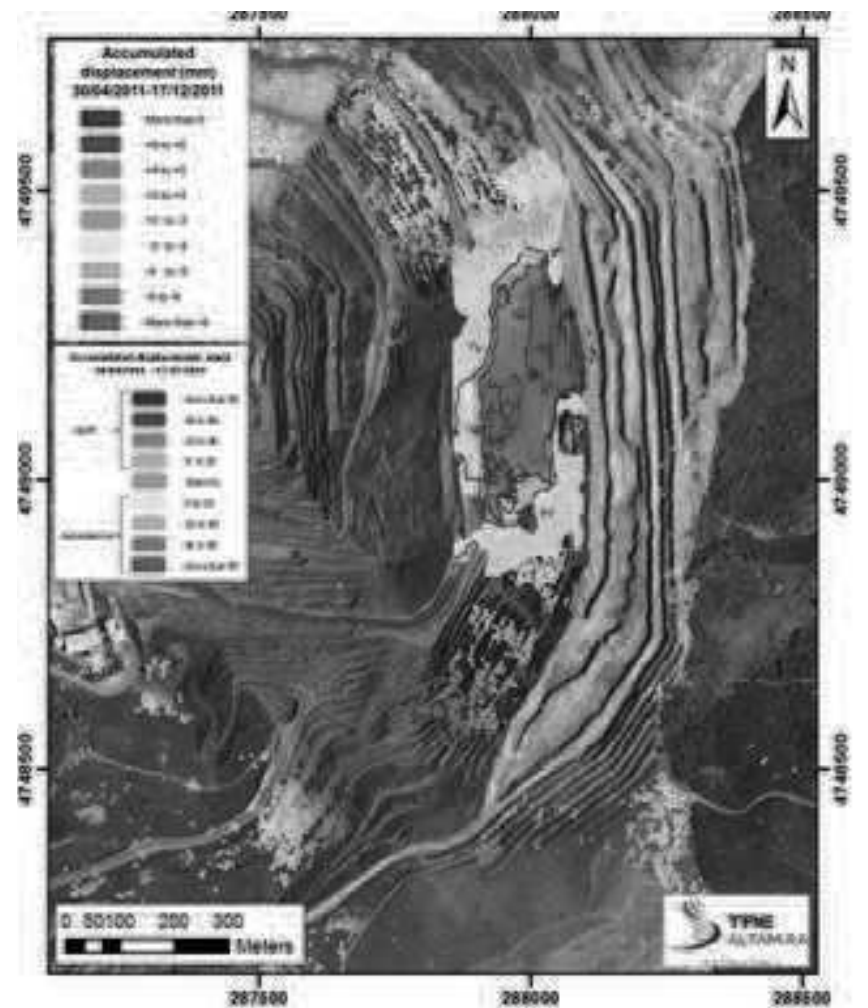

Carte de mouvement du sol 2011

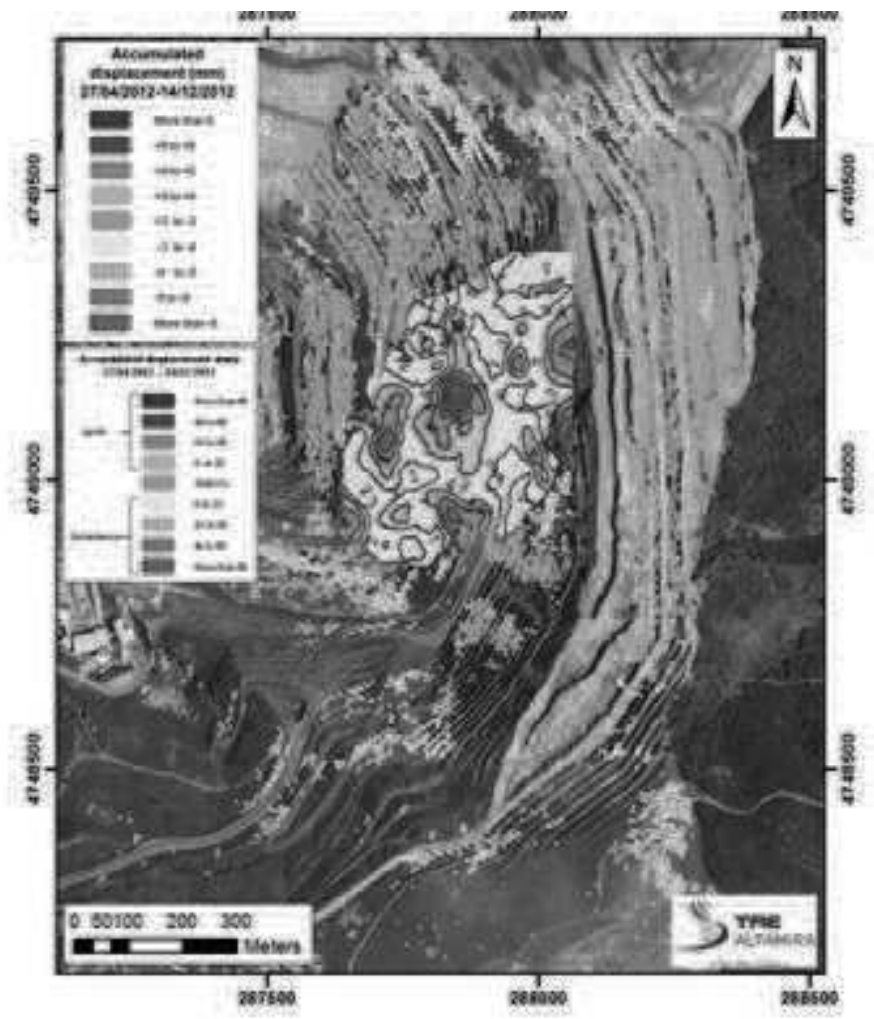

Carte de mouvement du sol 2012

Figure 7 : Exemple de la surveillance d'une mine de charbon qui combine exploitation souterraine et à ciel ouvert située en Espagne (Mora et al., 2013). Différentes techniques radar ont été combinées (méthode PSI et méthode basée sur les amplitudes des images SAR) pour rendre respectivement compte des déformations millimétriques mais aussi décimétriques à métriques qui affectent les talus actifs. Dans la surveillance menée sur ce site, (image gauche) l'étude a permis de déterminer le périmètre du talus affecté par les plus fortes déformations. L'inclinaison du talus a été modifiée l'année suivante (image de droite), ce qui se traduit par des vitesses moindres sur une zone d'influence plus large.
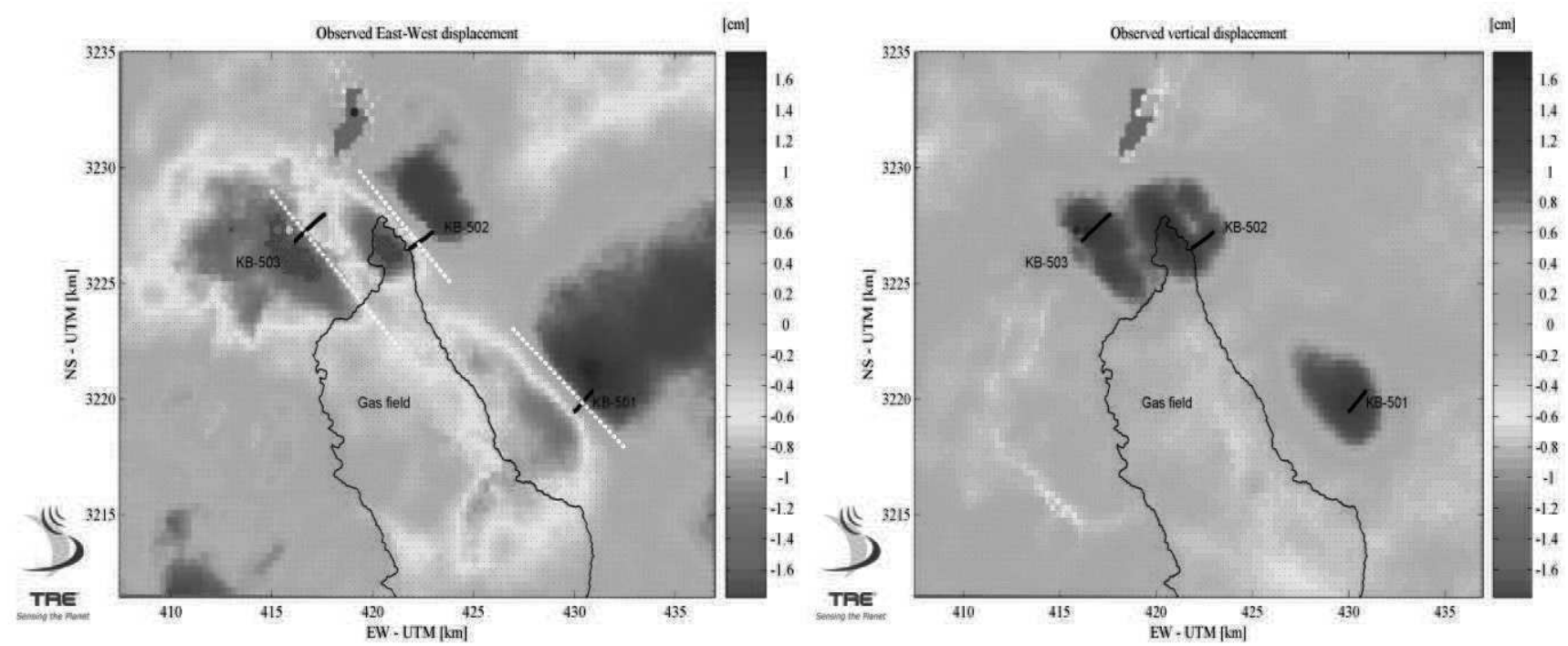

Figure 8 : Carte des déplacements sur le site In Salah de tests d'injection de $\mathrm{CO}_{2}$ entre 2004 et 2008. A gauche : composante horizontale Est-Ouest du déplacement. A droite : composante verticale du déplacement. Les traits noirs épais représentent l'étendue horizontale des trois puits d'injection dans le réservoir. D’après (Rucci et al., 2011). 


\section{Conclusion}

L'article a présenté quelques applications possibles de l'interférométrie SAR différentielle satellitaire pour le suivi de déformations d'origine anthropique, que ce soit en zone urbaine, ou hors zone urbaine pour des applications industrielles (mines, stockages de gaz).

Nous avons insisté sur la diversité des caractéristiques des phénomènes étudiés (allant de l'échelle du bâtiment à des extensions kilométriques) et sur les besoins en termes de surveillance ou de compréhension du phénomène. L'interférométrie SAR différentielle apparaît dans ce cadre comme un outil performant pour étudier ce type de déformation et comprendre les caractéristiques de sa source, qu'elle soit employée seule ou intégrée à des mesures au sol d'autre nature (e.g. mesures géodésiques ou géotechniques).

\section{Remerciements}

Les auteurs remercient TRE ALTAMIRA pour leur participation à l'article concernant les applications illustrées en figures 7 et 8 ainsi que les deux relecteurs pour leurs remarques pertinentes.

\section{Références}

Amelung F., Galloway D.L., Bell J.W., Zebker H.A., Laczniak R.J., 1999. Sensing the ups and downs of Las Vegas: InSAR reveals structural control of land subsidence and aquifer-system deformation. Geology, 27 (6), pp. 483-486.

Carnec C., Delacourt C., 2000. Three years of mining subsidence monitored by SAR interferometry, near Gardanne, France. Journal of Applied Geophysics, 43, pp. 43-54.

Chaussard E., Amelung F., Abidin H., Hong S.-H., 2013. Sinking cities in Indonesia: ALOS PALSAR detects rapid subsidence due to groundwater and gas extraction. Remote Sensing of Environment, 128, pp. 150-161.

Colesanti C., Le Mouelic S., Bennani M., Raucoules D., Carnec C., Ferretti A., 2005. Detection of mining related ground instabilities using the Permanent Scatterers technique - A case study in the East of France. International Journal of Remote Sensing, 26 (1), pp. 201-208.

Engelbrecht J., Inggs M.R., Makusha G., 2011. Detection and monitoring of surface subsidence associated with mining activities in the witbank coalfields, South Africa, using Differential Radar Interferometry. South African Journal of Geology, 114 (1), pp. 77-94.

Fruneau B., Rudant J.-P., Obert D., Raymond D., 1998. Small displacements detected by SAR interferometry on the city of Paris (France). Proc. of the 2nd International workshop on retrieval of bio- and geo-physical parameters from SAR data for land applications, 21-23
October 1998, Noordwijk, The Netherlands, pp. 557-563.

Fruneau B., Sarti F., 2000. Detection of ground subsidence on the city of Paris using Radar Interferometry: Isolation of deformation from atmospheric artifacts using correlation. Geophysical Research Letters, 28, pp. 3981-398.

Fruneau B., Deffontaines B., Prunier-Leparmentier A.M., Rudant J.-P., 2004. Suivi des déplacements verticaux par interférométrie radar : exemple de la gare Haussmann-Saint-Lazare (Paris). Revue Française de Géotechnique, 106-107, pp.103-110.

Guéguen Y., Deffontaines B., Fruneau B., Al Heib M., De Michele M., Raucoules D., Guise Y., Planchenault J., 2009. Monitoring residual mining subsidence of Nord/Pas-de-Calais coal basin from Differential and Persistent Scatterer Interferometry (Northern France). Journal of Applied Geophysics, 69, pp. 24-34.

Le Mouelic S., Raucoules D., Carnec C., King C., Adragna F., 2002. Ground uplift in the city of Paris (France) detected by satellite radar interferometry. Geophysical Research Letters, 29 (17), pp. 1853.

Loschetter A., Rohmer J., Raucoules D., de Michele M., 2015. Designing a ground-based geodetic measurement network for risk-oriented monitoring of surface deformations induced by $\mathrm{CO}_{2}$ deep injection. International Journal of Greenhouse Gas Control, 42, pp. 571-582.

Magalhaes S., 2010. L'interférométrie radar (DInSAR et PSI) appliquée à l'étude du risque mouvement de terrain à Paris. Thèse Université Paris-Est, $302 \mathrm{p}$.

Massonnet D., Feigl K.L., 1998. Radar interferometry and its application to changes in the Earth's surface. Reviews of Geophysics, 36 (4), pp. 441-500.

Mora 0., Álvarez I., Amor Herrera E., 2013. Slope stability monitoring in open pit and underground mine by means of Radar Interferometry, Slope Stability 2013, Brisbane Australia, 25-27 September 2013.

Nahli A., Simonetto E., Merrien-Soukatchoff V., Durand F., Rangeard D., 2018. Sentinel-1 for monitoring tunnel excavations in Rennes, France. SARWatch, 21-23 novembre 2018, Lisbonne, Portugal, Procedia Computer Science, 138, pp.393-400.

Onuma T., Ohkawa S., 2009. Detection of surface deformation related with $\mathrm{CO}_{2}$ injection by DInSAR at In Salah, Algeria. Energy Procedia, 92 (1), pp. 2177-2184.

Raucoules D., Le Mouélic S., Carnec C., Maisons C., King C., 2003. Urban subsidence in the city of Prato (Italy) monitored by radar satellite radar interferometry. International Journal of Remote Sensing, 24 (4), pp. 891-898.

Raucoules D., Colesanti C., Carnec C, 2007. Use of SAR interferometry for detecting and assessing ground subsidence. Compte Rendus Geosciences, 339 (5), pp. 289-302.

Raucoules D., Bourgine B., De Michele M., Le Cozannet G., Closset L., Bremmer C., Veldkamp H., Tragheim D., Bateson L., Crosetto M., Agudo M. Engdahl M, 2009. Validation and intercomparison of Persistent Scatterers 
Interferometry: PSIC4 project results. Journal of Applied Geophysics, 68, pp. 335-347.

Raucoules D., Le Cozannet G., Woppelmann G., De Michele M., Gravelle M., Daag A., Marcos M., 2013. High nonlinear urban ground motion in Manila (Philippines) from 1993 to 2010 observed by DInSAR: implications for sea level measurement. Remote Sensing of Environment, 139, pp. 386-397.

Rucci A., Vasco D.W., Novali F., 2011. Monitoring the geological storage of carbon dioxide using multicomponent SAR interferometry. Geophysical Journal International, 193 (1), 03 December 2011.

Rohmer J., Raucoules D., 2012. On the applicability of Persistent Scatterers interferometry (PSI) analysis for long term $\mathrm{CO}_{2}$ storage monitoring. Engineering Geology, pp. 147-148.

Simonetto E., Fruneau B., Raucoules D., 2019. Principes de l'interférométrie d'images radar pour la mesure de la topographie et des déplacements du sol et avancées récentes. RFPT, même numéro.

Strozzi T., Wegmüller U., 1999. Land subsidence in Mexico City mapped by ERS Differential SAR Interferometry. Int. Geoscience and Remote Sensing Symposium 1999, pp. 1940-1942.

Tesauro M., Berardino P., Lanari R., Sansosti E., Fornaro F., 2000. Urban subsidence inside the city of Napoli (Italy) observed by satellite radar interferometry. Geophysical Research Letters, 27, pp. 1961-1964.

Thierry P., Deverly F., Reppelin M., Simonetto E., Lembezat C., Arnaud A., Raucoules D., Closset L., King C., 2005. Survey of linear subsidence in an urban area using a $3 D$ geological model and satellite Differential InSAR. GEOLINE'05, Lyon, France.

Vasco D.W., Rucci A., Ferretti A., Novali F., Bissell R.C., Ringrose P.S., Mathieson A.S., Wright I.W., 2010. Satellite-based measurements of surface deformation reveal fluid flow associated with the geological storage of carbon dioxide. Geophysical Research Letters, 37, L03303.

Yan Y., Doin M.P., Lopez-Quiros P., Tupin F., Fruneau B., Pinel V., Trouvé E., 2012. Mexico City subsidence measured by InSAR time series: Joint analysis using PS and SBAS approaches. IEEE J. of Selected Topics in Applied Earth Observations and Remote Sensing, DOI:10.1109/JSTARS.2012.2191146.

Zebker H.A., Villasenor J., 1992. Decorrelation in Interferometric Radar Echoes. IEEE TGRS, 30 (5), pp. 950-959. 